\title{
SIMULAÇÃO CLÍNICA: EDUCAÇÃO PARA EQUIPE DE ENFERMAGEM NO CUIDADO AOS PACIENTES COM SONDA NASOENTERAL
}

\author{
CLINICAL SIMULATION: EDUCATION FOR \\ NURSING TEAM IN THE CARE OF PATIENTS \\ WITH NASOENTERAL TUBE
}

\section{SIMULACIÓN CLÍNICA: EDUCACIÓN PARA EL EQUIPO DE ENFERMERÍA EN EL CUIDADO DE PACIENTES CON SONDA NASOENTERAL}

\author{
Ana Paula Almeida Corrêa ${ }^{1}$ \\ Carlise Rigon Dalla Nora ${ }^{2}$ \\ Stella Marys Rigatti Silva ${ }^{3}$ \\ Graziela Lenz Viegas ${ }^{4}$ \\ Gabriele Peres Sousa ${ }^{5}$ \\ Mariur Gomes Beghetto ${ }^{6}$
}

Como citar este artigo: Corrêa APA, Nora CRD, Silva SMR, Viegas GL, Sousa GP, Beghetto MG. Simulação clínica: educação para equipe de Enfermagem no cuidado aos pacientes com sonda nasoenteral. Rev baiana enferm. 2021;35:e41998.

Objetivo: compreender como os técnicos de Enfermagem percebem a simulação clínica como metodologia de educação no cuidado ao paciente hospitalizado em uso de sonda nasoenteral. Método: trata-se de estudo exploratório, descritivo e de abordagem qualitativa, realizado com 64 técnicos de Enfermagem em um hospital de Porto Alegre, Sul do Brasil, em agosto e setembro de 2017. Realizou-se uma intervenção de educação por meio de simulações clínica, gravadas e transcritas. Utilizou-se a análise de conteúdo para tratamento dos dados. Resultados: foram estabelecidas duas categorias: as potencialidades da simulação clínica como metodologia de educação e os desafios da simulação clínica para a prática da educação. Conclusão: os profissionais de Enfermagem perceberam as potencialidades da atividade de simulação e mostraram-se colaborativos e receptivos a revisar as práticas de cuidados em terapia nutricional enteral. A simulação clínica é uma metodologia promissora a ser utilizada na educação permanente em serviço.

Descritores: Simulação. Treinamento por Simulação. Capacitação em Serviço. Educação em Enfermagem. Equipe de Enfermagem.

\footnotetext{
Enfermeira. Doutora em Enfermagem. Enfermeira do Serviço de Enfermagem Cirúrgica do Hospital de Clínicas de Porto Alegre. Porto Alegre, Rio Grande do Sul, Brasil. http://orcid.org/0000-000I-8890-1767.

2 Enfermeira. Doutora em Enfermagem. Professora da Universidade Federal do Rio Grande do Sul. Porto Alegre, Rio Grande do Sul, Brasil. carlise.nora@ufrgs.br. http://orcid.org/0000-000I-550I-2| 46 .

Enfermeira. Doutora em Enfermagem. Enfermeira do Centro de Pesquisa Clínica do Hospital de Clínicas de Porto Alegre. Porto Alegre, Rio Grande do Sul, Brasil. http://orcid.org/0000-0002-4124-519X.

4 Enfermeira. Enfermeira do Serviço de Enfermagem Cirúrgica do Hospital de Clínicas de Porto Alegre. Porto Alegre, Rio Grande do Sul, Brasil. http://orcid.org/0000$0001-7093-7470$.

5 Enfermeira. Universidade Federal do Rio Grande do Sul. Porto Alegre, Rio Grande do Sul, Brasil. http://orcid.org/0000-0002-9330-0234.

6 Enfermeira. Doutora em Epidemiologia. Professora da Universidade Federal do Rio Grande do Sul. Porto Alegre, Rio Grande do Sul, Brasil. http://orcid.org/00000002-9437-4999.
} 
Objective: to understand Nursing technicians'perception of clinical simulation as an education methodology in the care of hospitalized patients using nasoenteral tubes. Method: this is an exploratory, descriptive and qualitative study, conducted with 64 Nursing technicians from a hospital in Porto Alegre, Southern Brazil, in August and September 2017. An education intervention was carried out through clinical simulations, recorded and transcribed. Content analysis was used for data processing. Results: two categories were established: the potentialities of clinical simulation as an education methodology and the challenges of clinical simulation for the practice of education. Conclusion: Nursing professionals realized the potentialities of the simulation activity and were collaborative and receptive to review care practices in enteral nutritional therapy. Clinical simulation is a promising methodology to be used in continuing education in service.

Descriptors: Simulation Technique. Simulation Training. Inservice Training. Education, Nursing. Nursing, Team.

Objetivo: entender cómo los técnicos de enfermería perciben la simulación clínica como una metodología educativa en la atención de pacientes hospitalizados utilizando sondas nasoenterales. Método: se trata de un estudio exploratorio, descriptivo y cualitativo, realizado con 64 técnicos de enfermería en un hospital de Porto Alegre, sur de Brasil, en agosto y septiembre de 2017. Se llevó a cabo una intervención educativa a través de simulaciones clínicas, registradas y transcritas. El análisis de contenido se utilizó para el procesamiento de datos. Resultados: se establecieron dos categorias: las potencialidades de la simulación clinica como metodología educativa y los desafios de la simulación clínica para la práctica de la educación. Conclusión: los profesionales de enfermería percibieron las potencialidades de la actividad de simulación y fueron colaborativos y receptivos para revisar las prácticas de atención en la terapia nutricional enteral. La simulación clínica es una metodología prometedora que se utilizará en la educación continua en el servicio.

Descriptores: Simulación. Entrenamiento Simulado. Capacitación en Servicio. Educación en Enfermería. Grupo de Enfermería.

\section{Introdução}

Incidentes relacionados à Terapia Nutricional Enteral (TNE) podem estar diretamente ligados à inserção e manutenção da Sonda Nasoenteral (SNE), bem como pela administração de terapêutica pela sonda ${ }^{(1)}$. Nesse sentido, diferentes entidades, como a European Society for Clinical Nutrition and Metabolism (ESPEN) e a American Society for Parenteral and Enteral Nutrition (ASPEN), por meio de seus especialistas, estabeleceram diretrizes embasadas nas melhores evidências, a fim de manter a segurança do paciente que faz uso de $\mathrm{TNE}^{(2-3)}$.

A segurança do paciente em uso da TNE depende da vigilância e da avaliação constante da equipe de Enfermagem, que, por meio de competências específicas, possibilita o estabelecimento do cuidado nutricional e evita que ocorram incidentes relacionados ao processo de administração da dieta ${ }^{(4)}$. O desafio encontrado é o de adotar práticas seguras e exequíveis que contemplem todas as especificidades da administração da dieta por sonda. Ainda, deve-se considerar que somente o conhecimento é insuficiente para o desenvolvimento dessas práticas, uma vez que uma assistência segura depende das habilidades e do comprometimento dos profissionais $^{(4)}$.

Diferentes estratégias de educação para profissionais são utilizadas pelas instituições de saúde com foco na segurança do paciente, sendo uma delas, a metodologia ativa de ensino, conhecida como simulação clínica. Esta consiste em um processo dinâmico, que envolve a criação de um cenário fictício e representa, de forma autêntica, a realidade. Essa estratégia de ensino-aprendizagem facilita a participação ativa, integrando as complexidades da teoria e prática com oportunidades para repetição, feedback, avaliação e reflexão ${ }^{(5)}$.

Uma das vertentes do emprego da simulação para profissionais da área da saúde envolve o treinamento e visa o desenvolvimento de habilidades técnicas ${ }^{(6)}$. Achados na literatura demonstram que a simulação clínica vem sendo inserida no contexto dos profissionais de Enfermagem, com foco na segurança do paciente, seja para o desenvolvimento de habilidades para procedimentos inerentes ao papel do enfermeiro ${ }^{(7)}$ ou 
para o reconhecimento de sinais de deterioração clínica e eventos adversos ${ }^{(8)}$.

Nesse sentido, a simulação é capaz de replicar a prática clínica e contribuir para o aprendizado dos profissionais da área da saúde, possibilitando a adesão de habilidades não técnicas (cognitivas, sociais e pessoais), mas que complementam as habilidades técnicas, o que traz benefícios para o cuidado ao paciente, contribuindo para sua segurança, para o desempenho eficaz das tarefas ${ }^{(9)}$ e para o desenvolvimento do trabalho interprofissional ${ }^{(10)}$. Diante dessa premissa, o uso da simulação permite que o profissional torne-se um participante na construção de seu conhecimento e não um mero receptor passivo, assumindo uma condição de coparticipante no desenvolvimento de sua prática clínica ${ }^{(11)}$.

$\mathrm{Na}$ Enfermagem, a simulação é apontada como uma metodologia favorável ao desenvolvimento de postura ética no processo de trabalho $^{(12)}$. Existem evidências de que a simulação gera diversas competências e habilidades relevantes para o exercício profissional, como a tomada de decisão, a empatia com a profissão, a articulação entre teoria e prática, o desenvolvimento da liderança e a melhoria nos processos de trabalho dos serviços de saúde com consequente diminuição dos erros ${ }^{(13)}$.

A pesquisa foi direcionada para os técnicos de Enfermagem, pois compete à equipe multiprofissional que atua na TNE participar dos treinamentos sobre as boas práticas em TNE, com vista a manter a assistência segura. Assim, é fundamental potencializar estratégias que contribuam para a educação dos técnicos de Enfermagem, os processos de trabalho e a segurança e qualidade no cuidado com o paciente. Nesse contexto, este estudo tem como objetivo compreender como os técnicos de Enfermagem percebem a simulação clínica como metodologia de educação no cuidado ao paciente hospitalizado em uso de sonda nasoenteral.

\section{Método}

Trata-se de estudo exploratório, descritivo e de abordagem qualitativa, que teve origem em um ensaio clínico intitulado: "Efeito de uma intervenção de simulação clínica sobre as práticas de técnicos de Enfermagem no cuidado ao paciente em uso de sonda nasoenteral: ensaio clínico." O presente estudo analisou um recorte dos dados qualitativos que se referem à percepção dos técnicos de Enfermagem sobre a simulação clínica no cuidado ao paciente em uso da sonda nasoenteral durante a intervenção do ensaio clínico.

O estudo foi realizado nas unidades de internação para adultos de um hospital de grande porte da cidade de Porto Alegre, Sul do Brasil. Participaram do estudo 64 técnicos de Enfermagem das áreas de internação clínica e cirúrgica do hospital. Os critérios usados foram: ser técnico de Enfermagem e atuar nas unidades de internação clínica e cirúrgica onde ocorreu a intervenção. Foram excluídos do estudo técnicos de Enfermagem em gozo de férias ou em licença para tratamento de saúde no período de coleta dos dados. Para este estudo foram potencialmente elegíveis todas as falas dos técnicos de Enfermagem que participaram da etapa de intervenção (simulação clínica) do estudo matriz, sendo considerada uma amostra intencional, e excluídos os que estavam afastados do trabalho durante o período de coleta de dados.

O processo de coleta de dados seguiu a diretriz das atividades de simulação ${ }^{(14)}$. Esta intervenção foi fundamentada nos Protocolos Operacionais Padrão (POPs) institucionais para assistência ao paciente em uso de sonda nasoenteral e nas diretrizes de Boas Práticas em Terapia Nutricional Enteral $^{(2-3)}$ e da European Society for Clinical Nutrition and Metabolism ${ }^{(15)}$. A coleta de dados foi realizada em agosto e setembro de 2017.

Para a realização do cenário de simulação clínica utilizou-se um manequim de baixa fidelidade, no qual os técnicos de Enfermagem deveriam identificar e corrigir os erros na assistência ao paciente em uso de SNE, tais como: a inconsistência entre a identificação do paciente e o rótulo de dieta, a administração da dieta com cabeceira baixa, a fixação da sonda descolada e suja, o atraso na administração da dieta, a data vencida do equipo de dieta enteral, o resíduo de 
dieta na seringa e o copo plástico utilizado para higienização da sonda e equipo, além da não identificação desses utensílios. A duração dos cenários foi cerca de 30 a 45 minutos durante o turno de trabalho.

A simulação seguiu um roteiro que consistia em sete itens: o primeiro refere-se ao nome do cenário: "Simulação clínica dos cuidados com a sonda nasoenteral." O segundo item é o Briefing (momento da apresentação do cenário e orientação para os técnicos de Enfermagem). O enfermeiro facilitador, após realizada a leitura do caso, salientava que o Técnico Atuante (TA) deveria agir como se estivesse em situação de atendimento ao paciente que utilizava SNE, realizando e relatando as modificações necessárias no cenário. Já o Técnico Observador (TO) deveria fazer silêncio e não interferir na cena.

O terceiro item do roteiro aborda os objetivos da simulação: identificar as não conformidades em relação aos cuidados com a SNE, relatar e/ou executar os cuidados adequados para corrigir as não conformidades encontradas e recordar as rotinas corretas quanto ao uso da SNE. O quarto item trata dos materiais necessários para a manutenção da terapia: manequim de baixa fidelidade, maca, sonda nasoenteral, pulseira de identificação do paciente, bomba de infusão enteral, equipo enteral, seringa Oralpak ${ }^{\circledR} 10 \mathrm{ml}$, copo plástico descartável, frascos de dieta enteral, frascos de água para hidratação, frascos de água para higienização de dispositivos, fita métrica, estetoscópio, rótulos de identificação de frascos de dieta, de águas e de dispositivos (seringas, equipos e copos plásticos), esparadrapo, micropore, almotolia de álcool, prescrição médica e de enfermagem, folha de registro de sinais vitais e monitorização hídrica, prancheta e caneta.

O item cinco refere-se aos participantes do cenário da simulação: um técnico de Enfermagem atuante no cenário, um técnico de Enfermagem observador do cenário, um pesquisador facilitador da simulação e um pesquisador coletor dos dados (ambos enfermeiros). O sexto item refere-se ao ambiente onde ocorreu a simulação clínica: uma sala contígua à área assistencial, com um manequim posicionado em uma maca em decúbito dorsal e uma cabeceira totalmente reta, recebendo dieta por SNE. Algumas não conformidades eram apresentadas no cenário e esperava-se que o técnico de Enfermagem as identificasse, executando-as ou relatando como seria a correta execução. Alguns exemplos dessas não conformidades: o manequim estava recebendo dieta com a cabeceira baixa, em decúbito dorsal plano, a fixação da SNE estava com oleosidade, sujidade e pouco descolada, a dieta infundida estava vencida, a água de hidratação estava identificada com nome de outro paciente e os dispositivos para higienização do equipo e sonda não estavam rotulados e apresentavam sujidade de dieta.

O sétimo item do roteiro é o Debriefing, momento que visa refletir e revisar as rotinas assistenciais para os cuidados com a SNE. O instrutor foi o facilitador, realizando as seguintes perguntas ao técnico de Enfermagem que atuou no cenário: Como você descreve o cenário? Como o manequim foi encontrado? Você identificou alguma não conformidade? O que você fez durante o atendimento? Como procedeu durante o atendimento? O que você fez que considera correto? Como se sentiu no atendimento? O objetivo proposto foi atingido? O que você faria diferente no atendimento? O que você relembrou com essa experiência?

Foram realizadas trinta simulações, em que todos os áudios foram gravados e, em seguida, foi realizada a transcrição dos dados. Depois da leitura de todas as transcrições, foram consideradas para este estudo as falas referentes às respostas dadas às perguntas: "Como se sentiu no atendimento?" e "O que você relembrou com essa experiência?".

Destaca-se ainda que as falas dos participantes podem se referir ao momento do cenário propriamente dito ou ao momento do debriefing, em que houve a participação de ambos os técnicos (atuantes e observadores).

A análise dos dados seguiu a orientação da Análise de Conteúdo do tipo temática, constituída de três etapas: Pré-análise: corresponde à fase de transcrição e organização dos dados, em que se retomaram os objetivos da pesquisa 
com o intuito de operacionalizar e sistematizar as ideias iniciais; Exploração do material: foram definidas e organizadas as categorias temáticas, iniciadas na fase anterior; Tratamento dos resultados e interpretação: os dados do estudo foram articulados com a literatura da área e construídas novas informações com base no objeto de estudo ${ }^{(16)}$.

O estudo seguiu as recomendações do Consolidated criteria for reporting qualitative research (COREQ) $)^{(17)}$. O estudo matriz foi registrado no Clinical Trials (NCT03497221) e aprovado em seus aspectos éticos e metodológicos pelo Comitê de Ética em Pesquisa (CEP) da instituição (CAAE n. 63247916500005327). Todos os participantes assinaram o Termo de Consentimento Livre e Esclarecido (TCLE). O anonimato dos participantes foi garantido com a identificação dos participantes pelas letras "a" de técnico atuante e "o" de técnico observador, acompanhada do número correspondente à ordem de transcrição da simulação clínica.

\section{Resultados}

Do total de 87 técnicos de Enfermagem que atuam nas unidades clínicas e nas unidades cirúrgicas, 64 (73,6\%) participaram da simulação. Desses, 30 (46,9\%) desempenharam a função de "atuantes", os demais desempenharam a função de "observadores". Entre os participantes, 33 (51,6\%) trabalhavam na unidade clínica e 31 (48,4\%) na unidade cirúrgica. A maioria dos participantes $(84,4 \%)$ eram mulheres, com média de 6 (4-15) anos de trabalho na instituição. Quanto ao turno de trabalho, 28,1\% trabalhavam pela manhã, 32,8\% à tarde e 39,1\% à noite. Ao serem questionados, 38 (59,4\%) relataram que realizaram algum tipo de treinamento ou capacitação institucional para administração de TNE, enquanto 17 (26,6\%) disseram nunca ter participado desse tipo de atividade, e os demais, 9 (14,1\%), não lembravam ou não informaram.

O processo de análise dos dados resultou na construção de duas categorias temáticas: potencialidades da simulação clínica como metodologia de educação e os desafios da simulação clínica para a prática da educação.

\section{Potencialidades da simulação clínica como metodologia de educação}

Esta categoria apresenta os aspectos positivos atribuídos pela equipe de Enfermagem à prática de simulação clínica no serviço de saúde. Nesse sentido, os participantes, em sua maioria, indicaram que o cenário utilizado representou a realidade vivenciada no dia a dia e indicaram a compreensão da simulação como metodologia de aprendizagem no serviço, capaz de romper com a "automatização do cuidado", além de expressarem uma percepção positiva da prática.

Dessa forma, nas falas dos participantes é possível identificar relatos sobre o realismo do cenário da simulação clínica:

\footnotetext{
Esse cenário montado se aproxima da nossa realidade. Eu acho que nós temos que cuidar, temos que ver direitinho como instalar e testar tudo [...]. (a14).

É, é real a cena. (a3).

Sim, bastante real. (a7).

Tem relação com a realidade. (a15).

O que foi encontrado na cena é semelhante com o que encontramos [...] Nossa, isso é totalmente real [...]. (a5).

Esse cenário montado se aproxima da nossa realidade. (a14).
}

Para os participantes, a simulação colaborou com a revisão e a reflexão sobre suas práticas, na adesão de conhecimento e evitou possíveis riscos aos pacientes que fazem uso de TNE.

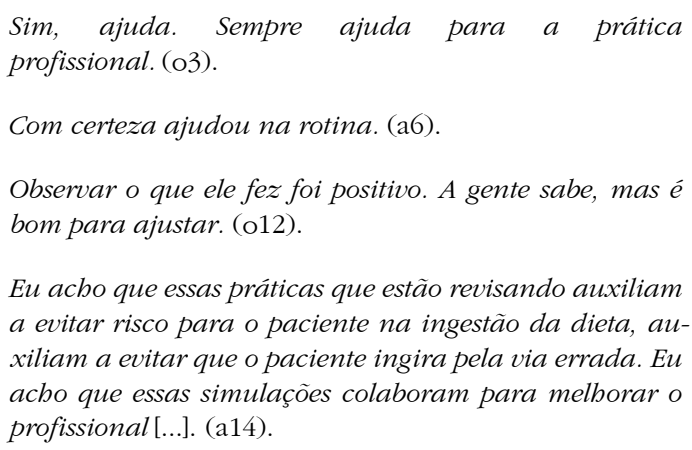

Eu acho que essas práticas que estão revisando auxiliam a evitar risco para o paciente na ingestão da dieta, auxiliam a evitar que o paciente ingira pela via errada. Eu acho que essas simulações colaboram para melhorar o profissional [...]. (a14).

Nossa, ajudou! Porque para tudo que a gente aprende, a gente não esquece, eu acho que tem que botar sempre em prática. (a14). 
Da mesma forma, as falas dos participantes indicaram que a simulação foi útil para romper com a "automatização do cuidado":

Toda capacitação, mesmo que faça parte de nossa rotina, é sempre importante. É que, às vezes, mesmo com muito tempo de prática, a gente acaba ficando um pouco relapso. (o13).

Com certeza ajudou a melhorar! São coisas que as pessoas vão esquecendo ou deixando de fazer [...]. (a15).

[...] talvez por ser muito corrido, tu não observe, mas sobre os cuidados de outros olbos, tu te preocupa mais [...]

Será que eu fiz tudo certo como eu faço geralmente? (a21).

Assim, percebe-se que os participantes expressaram um feedback positivo sobre a simulação clínica realizada no ambiente de trabalho, conforme as falas a seguir:

Eu achei bem bom. (o7).

Tudo é válido. Eu gostei! (o10).

Bom. É bom, né?(a11).

Sempre é bom fazer isso aqui, né? (a12).

Ajudou muito! (o21).

\section{Desafios da simulação clínica para prática da educação}

Esta categoria temática abordou elementos que podem ser considerados desafiadores para a prática da simulação clínica. São elementos relativos ao nervosismo por participar da simulação, o desconforto diante do fato de avaliar o colega e/ou de identificar não conformidades no cenário.

Percebeu-se um certo nervosismo dos participantes ao participar/atuar na simulação clínica, conforme as falas a seguir:

Eu fiquei um pouco nervosa. (a10).

Nervosa, né? São coisas que a gente faz no dia a dia, mas tu cria uma rotina, né? (a17).

Sim, a gente fica um pouco ansiosa de estar sendo observada [...]. (a6).

Da mesma forma, alguns participantes sentiram-se desconfortáveis ao avaliar o colega ou devido à proposta de identificar não conformidades no cenário, de acordo com as falas:

A simulação que a gente está fazendo é ruim, a gente está avaliando o colega. (a21).

\section{As pegadinhas é que foram o problema. (a11).}

Em síntese, quando comparamos as categorias potencialidades e os desafios da simulação, fica evidente que esta última apresentou um menor número de falas nas categorias temáticas.

\section{Discussão}

A aproximação do cenário com o ambiente real foi citada pelos técnicos de Enfermagem como uma das principais potencialidades da simulação clínica para a equipe de Enfermagem. Estudo de revisão ${ }^{(18)}$ revela que o realismo das simulações pode trazer benefícios e sucesso à atividade, pois leva os participantes a encararem a estratégia como legítima. O fato de desenvolver uma simulação clínica fidedigna à realidade em um cenário montado em uma sala contígua à área assistencial e com os mesmos utensílios utilizados para administração e manutenção da terapia nutricional enteral pode ter aproximado a experiência ao ambiente real, tornando a simulação autêntica e com elevado nível de realismo. O realismo do cenário provoca no indivíduo as mesmas respostas psicológicas que teria em um ambiente real de cuidado, o que leva o participante a desenvolver o pensamento crítico e as habilidades para a tomada de decisão ${ }^{(18)}$.

Nesse sentido, os resultados demonstram que a simulação clínica foi uma estratégia que auxiliou na revisão de práticas de cuidado e na adesão de conhecimentos em TNE. Um estudo $^{(19)}$ corrobora estes resultados ao demonstrar que a simulação clínica emerge como uma metodologia profícua, capaz de desenvolver habilidades técnicas e de comunicação entre os profissionais da saúde, proporcionando um espaço educativo que possibilita a reflexão acerca do processo de trabalho e identificando o que precisa ser transformado.

O relato de experiência, que envolveu as etapas de formação dos facilitadores, construção de cenários, desenvolvimento da simulação realística com a Enfermagem de um hospital de ensino na região Sul do Brasil, demonstrou que a simulação é uma metodologia que permite o 
processo de ensino-aprendizagem e pode ser amplamente utilizada para educação permanente dos profissionais de forma bem-sucedida ${ }^{(20)}$. Um estudo, que revisou 53 publicações sobre simulação clínica, evidenciou ganhos, como o aprimoramento do conhecimento e a capacidade de reflexão e pensamento crítico ${ }^{(18)}$.

A metodologia empregada na simulação do presente estudo utilizou o cenário com algumas não conformidades nos cuidados em TNE, semelhante a estudo realizado com estudantes da área da saúde, cujo objetivo foi evidenciar a melhor tomada de decisão, o que permitiu aos participantes a percepção dos erros cometidos durante a atuação $^{(21)}$. Os resultados do presente estudo demonstram que o cenário elaborado permitiu aos participantes a revisão de suas práticas e a reflexão acerca do próprio desempenho durante o cenário, avaliando como positiva a simulação, vista como uma oportunidade de melhoria $\mathrm{e}$ aprimoramento no processo de trabalho.

De mesma forma, os técnicos de Enfermagem consideraram que o processo de simulação auxiliou na reflexão acerca das questões de segurança do paciente e relataram que os cuidados poderiam evitar eventos adversos relacionados ao cuidado em TNE. A literatura demonstra que o cenário permite simular ações do cotidiano em um ambiente livre de riscos e alinhado com as boas práticas de Enfermagem ${ }^{(5)}$. Nesse sentido, as instituições, ao integrarem a simulação nos processos de educação em serviço focados na segurança do paciente, podem reduzir a ocorrência de eventos adversos acidentais ou evitáveis provocados pela equipe de saúde ${ }^{(20)}$.

Vale ressaltar ainda que a simulação apresenta vantagens para a segurança do paciente, para o desenvolvimento do trabalho em equipe e para a redução de custos reais, além de ser reconhecida como uma aliada na preparação da equipe de Enfermagem para o desempenho de habilidades técnicas e não técnicas no ambiente de trabalho. Uma parcela dos eventos adversos que ocorre nos serviços de saúde é atribuída ao não cumprimento dessas habilidades no ambiente de trabalho ${ }^{(9)}$.
Os resultados são inquietantes por indicarem que, por vezes, os técnicos de Enfermagem realizam as ações de forma automatizada. A obrigação legal e moral da equipe de saúde é realizar uma assistência de excelência ao paciente, em que o cuidado deve ser executado por meio da técnica correta e de forma integral ${ }^{(12)}$. Sendo assim, é preciso articular estratégias com base em evidências e normas que visem o cuidado seguro na assistência prestada ao paciente ${ }^{(22)}$.

Outro estudo de revisão ${ }^{(18)}$ corrobora $\mathrm{O}$ feedback positivo dos participantes deste estudo ao refletir e relatar os ganhos obtidos pelos profissionais da saúde durante o cenário de simulação, como: satisfação, autoconfiança, conhecimento, empatia, realismo, comunicação, motivação, capacidade de reflexão, pensamento crítico e trabalho em equipe. Neste estudo, itens como comunicação, trabalho em equipe e motivação não foram evidenciados.

O sentimento de nervosismo referido pelos participantes em relação à atuação foi considerado um desafio. Ensaio clínico randomizado ${ }^{(23)}$ realizado com 52 discentes de Enfermagem corrobora os achados ao apontar que a simulação indica que a ansiedade influencia no comportamento durante a execução do cenário e, por vezes, pode levar a um desempenho ruim quando os participantes se veem em situações de atendimento em emergências. Nesse sentido, a ansiedade é uma reação natural e pode ser gerada pelo sentimento de falta de preparo por parte dos técnicos de Enfermagem. Estudos referem que a falta de conhecimento e de habilidade estão entre as principais fontes de ansiedade, assim como o fato de estar sendo observado ou de sentir-se inexperiente em algum quesito avaliado na simulação ${ }^{(18,24)}$.

Foi relatado pelos participantes desconforto em avaliar o colega. É importante destacar que a forma como o instrutor conduz o momento da simulação é decisivo para determinar se a experiência será positiva ou negativa para os participantes $^{(25)}$. Nessa perspectiva, o debriefing é o momento chave da simulação, pois é quando se observa a conduta, reflete-se sobre ela e se compreende o que está faltando para 
que a competência desejada seja alcançada ${ }^{(25)}$. O debriefing oferece uma oportunidade aos participantes de revisarem seus conhecimentos, avaliarem as justificativas e as respostas às suas intervenções e desenvolverem habilidades ${ }^{(26)}$.

Este estudo apresentou algumas limitações referente à falta de experiência prévia com a metodologia de simulação por parte dos técnicos de Enfermagem, sendo, para muitos, o primeiro contato com esse tipo de metodologia de aprendizagem. O desenho do estudo não permite a generalização dos resultados, pois sua utilização precisa ser contextualizada aos profissionais da saúde inseridos em unidades de internação clínica e cirúrgica hospitalar, o que inviabiliza a generalização dos dados. Entretanto, algumas recomendações referentes ao fortalecimento da metodologia de simulação podem ser aplicáveis em outros contextos de cuidado. Assim, é necessário que novos estudos com profissionais que atuam na prática clínica sejam realizados por meio da metodologia de simulação, com vistas à melhoria da qualidade do cuidado e o desenvolvimento de estudos práticos baseados em evidências.

\section{Conclusão}

Os resultados demonstraram que a simulação clínica foi compreendida pelos técnicos de Enfermagem como uma metodologia que se aproxima da realidade do serviço, auxilia no aprendizado e permite utilizar o conhecimento prévio para revisar e prevenir eventos que possam causar danos ao paciente, além de ser uma oportunidade de refletir sobre o cuidado realizado. Os participantes deste estudo consideraram ainda que a simulação gerou nervosismo e desconforto ao avaliar o colega atuante no cenário.

Este estudo contribuiu para a compreensão da simulação enquanto metodologia para educação permanente no serviço, que pode colaborar para a aquisição de competências e habilidades e refletir em melhor desempenho dos técnicos de Enfermagem frente às questões de segurança do paciente. Assim, este estudo oferece subsídios para que os serviços de saúde invistam em metodologias ativas de aprendizado, como a simulação para seus profissionais. Da mesma forma, este estudo pode contribuir para a formação em saúde, na medida em que aponta a necessidade de utilizar essa metodologia durante a graduação em Enfermagem, permitindo o desenvolvimento de competências técnicas e não técnicas.

Não obstante não ser possível extrapolar os resultados para outras realidades, a compreensão da percepção dos técnicos de Enfermagem sobre a simulação clínica durante o cuidado ao paciente permite concluir que esta metodologia tem potencialidade e que é necessário continuar investigando essa prática de ensino nos diferentes contextos de saúde e com diferentes profissionais, para produzir mudanças positivas nos serviços de saúde.

\section{Colaborações}

1 - concepção, projeto, análise e interpretação dos dados: Ana Paula Almeida Corrêa e Stella Marys Rigatti Silva;

2 - redação do artigo e revisão crítica relevante do conteúdo intelectual: Ana Paula Almeida Corrêa, Carlise Rigon Dalla Nora, Stella Marys Rigatti Silva, Graziela Lenz Viegas e Gabriele Peres Sousa;

3 - aprovação final da versão a ser publicada: Carlise Rigon Dalla Nora e Mariur Gomes Beghetto.

\section{Referências}

1. Cervo AS, Magnago TSBS, Carollo JB, Chagas BP, Oliveira AS, Urbanetto JS. Adverse events related to the use of enteral nutritional therapy. Rev Gaúcha Enferm. 2014;35(2):53-9. DOI: $10.1590 / 1983-1447.2014 .02 .42396$

2. Mueller C, Compher C, Ellen DM. A.S.P.E.N. Clinical guidelines: Nutrition screening, assessment, and intervention in adults. J Parenter Enter Nutr. 2011;35(1):16-24. DOI: $10.1177 / 0148607110389335$

3. Druyan ME, Compher C, Boullata JI, Braunschweig CL, George DE, Simpser E, et al. Clinical Guidelines for the Use of Parenteral 
and Enteral Nutrition in Adult and Pediatric Patients: applying the GRADE system to development of A.S.P.E.N. clinical guidelines. J Parenter Enter Nutr. 2012;36(1):77-80. DOI: $10.1177 / 0148607111420157$

4. Leigh G, Stueben F, Harrington D, Hetherman S. Making the Case for Simulation-Based Assessments to Overcome the Challenges in Evaluating Clinical Competency. Int J Nurs Educ Scholarsh. 2016;13(1):27-34. DOI: 10.1515/ ijnes-2015-0048

5. Cogo ALP, Lopes EFS, Perdomini FRI, Flores GE, Rosa MRR. Building and developing realistic simulation scenarios on safe drug administration. Rev Gaúcha Enferm. 2019; 40 (spe):e20180175. DOI: 10.1590/1983-1447.2019.20180175

6. Sadideen H, Goutos I, Kneebone R. Burns education: The emerging role of simulation for training healthcare professionals. Burns. 2017;43(1):34-40. DOI: 10.1016/j.burns.2016.07.012

7. Farra S, Smith S, French DA, Gillespie G. Development of an assessment instrument to evaluate performance of the skill of decontamination. Nurse Educ Today. 2015;35(10):1016-22. DOI: 10.1016/j.nedt.2015. 04.010

8. Elder L. Simulation: A Tool to Assist Nursing Professional Development Practitioners to Help Nurses to Better Recognize Early Signs of Clinical Deterioration of Patients. J Nurses Prof Dev. 2017;33(3):127-30. DOI: 10.1097/ NND.0000000000000342

9. Carvalho EC. A glance at the non-technical skills of nurses: simulation contributions. Rev Latino-Am Enfermagem. 2016;24:e2791. DOI: $10.1590 / 1518-8345.0000 .2791$

10. Watters C, Reedy G, Ross A, Morgan NJ, Handslip R, Jaye P. Does interprofessional simulation increase self-efficacy: a comparative study. BMJ Open. 2015;13(5):e005472. DOI: 10.1136/bmjopen-2014-005472

11. Jerônimo IRL, Campos JF, Peixoto MAP, Brandão MAG. Use of clinical simulation to improve diagnostic reasoning in nursing. Esc Anna Nery. 2018;22(3):e20170442. DOI: 10.1590/2177-9465-ean-2017-0442

12. Oliveira Costa RR, Medeiros SM, Amado Martins JC, Dias Coutinho VR. Simulation in training nurses: reflections and justifications based on bioethics and human rights approaches. Acta bioeth. 2018;24(1):31-8. DOI:10.4067/ S1726-569X2018000100031

13. Nunes de Oliveira S, Prado ML, Silveira Kempfer S, Waterkemper R, Caravaca Morera JA, Bernardi MC. Learning through clinical experience simulation: perceptions in nursing students. Rev iberoam educ invest enferm. 2015;5(3):56-63. DOI: https://www. enfermeria21.com/revistas/aladefe/articulo/173/

14. Quilici AP, Abrão K, Timerman S, Gutierrez F. Simulação Clínica do Conceito à Aplicabilidade. Rio de Janeiro: Atheneu; 2012.

15. Schütz T, Herbst B, Koller M. Methodology for the development of the ESPEN Guidelines on Enteral Nutrition. Clin Nutr. 2006;25(2):203-9. DOI: 10.1016/j.clnu.2006.01.008

16. Minayo MCS. O desafio do conhecimento científico: pesquisa qualitativa em saúde. São Paulo: Hucitec; 2014.

17. Tong A, Sainsbury P, Craig J. Consolidated criteria for reporting qualitative research (COREQ): a 32 item checklist for interviews and focus groups. Ins J Qual Health Care. 2007;19(6):349-57. DOI: 10.1093/intqhe/mzm042 19

18. Negri EC, Mazzo A, Martins JCA, Pereira Junior GA, Pedersoli CE. Clinical simulation with dramatization: gains perceived by students and health professionals. Rev Latino-Am Enfermagem. 2017;25:e2916. DOI: 10.1590/1518-8345.1807.2916

19. Miranda FBG, Mazzo A, Pereira Junior GA. Assessment of individual and interprofessional skills of health professionals in simulated clinical activities: a scoping review. Interface (Botucatu). 2018;22(67):1221-34. DOI: 10.1590/ $1807-57622017.0628$

20. Groves PS, Bunch JL, Cram E, Perkhounkova Y. Development and feasibility testing of a patient safety research simulation. Clin Simul Nurs. 2018;15:27-33. DOI: 10.1016/j.ecns.2017.09.007

21. Presado MHCV, Colaço S, Rafael H, Baixinho CL, Félix I, Saraiva C, et al. Learning with High Fidelity Simulation. Ciênc Saúde Colet. 2018;23(1):51-9. DOI: 10.1590/1413-81232018231. 23072017

22. Magalhães AMM, Dall'Agnol CM, Marck PB. Nursing workload and patient safety - a mixed method study with an ecological restorative approach. Rev Latino-Am Enfermagem. 2013;21(spe):146-54. DOI: 10.1590/S0104-116920 13000700019 
23. Boostel R, Felix JVC, Bortolato-Major C, Pedrolo E, Vayego SA, Mantovanil MF. Stress of nursing students in clinical simulation: a randomized clinical trial. Rev Bras Enferm. 2018;71(3):1029-37. DOI: 10.1590/0034-71672017-0187

24. Finan E, Bismilla Z, Whyte HE, Leblanc V, Mcnamara PJ. High-fidelity simulator technology may not be superior to traditional low-fidelity equipment for neonatal resuscitation training. J Perinatol. 2012;32(4):287-92. DOI: 10.1038/ jp.2011.96

25. Oliveira SN, Massaroli A, Martini JG, Rodrigues J. From theory to practice, operating the clinical simulation in Nursing teaching. Rev Bras Enferm. 2018;71(Suppl 4):1791-98. DOI: 10.1590/0034-7167-2017-0180

26. Bortolato-Major C, Mantovani MF, Felix JVC, Boostel R, Silva ÂTM, Caravaca-Morera JA. Avaliação do debriefing na simulação clínica em enfermagem: um estudo transversal. Rev Bras Enferm. 2019;72(3):825-31. DOI: https://doi. org/10.1590/0034-7167-2018-0103

Recebido: 7 de outubro de 2020

Aprovado: 19 de janeiro de 2020

Publicado: 5 de abril de 2021

A Revista Baiana de Enfermagem utiliza a Licença Creative Commons - Atribuição-NãoComercial 4.0 Internacional.

https://creativecommons.org/licenses/by-nc/4.0/

Este artigo é de acesso aberto distribuído sob os termos da Licença Creative Commons (CC BY-NC).

Esta licença permite que outros remixem, adaptem e criem a partir do seu trabalho para fins não comerciais. Embora os novos trabalhos tenham de lhe atribuir o devido crédito e não possam ser usados para fins comerciais, os usuários não têm de licenciar esses trabalhos derivados sob os mesmos termos. 